\title{
APLICAÇÃO FOLIAR DE NITROGÊNIO EM VIDEIRA: AVALIAÇÃO DO TEOR NA FOLHA E DAS RESERVAS NITROGENADAS E DE CARBOIDRATOS NAS GEMAS DOS RAMOS DO ANO ${ }^{1}$
}

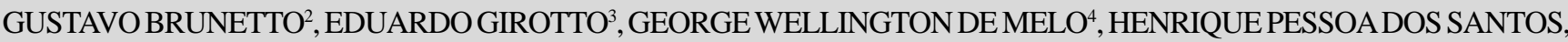 \\ CARLOS ALBERTO CERETTA ${ }^{5}$, JOÃO KAMINSKI ${ }^{5}$, RENAN COSTA BEBER VIEIRA $^{6}$
}

RESUMO - No Rio Grande do Sul (RS), as aplicações foliares de nitrogênio, quando necessárias, têm sido usadas para complementar a adubação via solo. Entretanto, carece-se de informações dos efeitos da freqüência e da quantidade de $\mathrm{N}$ aplicado sobre a sua dinâmica na folha e de reservas nitrogenadas e de carboidratos nas partes perenes da videira, que compõem o objetivo deste trabalho. O trabalho foi conduzido em um vinhedo da cultivar Chenin Blanc, safra 2004/05, na Embrapa Uva e Vinho, em Bento Gonçalves (RS), sobre um Neossolo Litólico. Os tratamentos consistiram de uma, duas e três aplicações foliares de 0 (água); 1,$11 ; 2,23 ; 3,31$ e 4,41g de $\mathrm{N}$ planta $^{-1}$. Após cada aplicação de nitrogênio, foram coletadas folhas inteiras (limbo+pecíolo) no terço médio dos ramos do ano, no interior e exterior dos diferentes lados da planta, secas, moídas e preparadas para a análise de $\mathrm{N}$ total. Na última época de coleta de folhas, foram coletados três ramos do ano em cada planta, retiradas seis gemas em cada ramo, as quais foram submetidas à análise de amido, carboidratos solúveis totais, carboidratos redutores, aminoácidos totais e proteínas totais. As aplicações foliares de $\mathrm{N}$ aumentaram o teor do nutriente na folha inteira, de forma destacada, nas épocas de coletas próximas às aplicações; entretanto, essas aplicações diminuíram os teores de amido e carboidratos solúveis totais nas gemas dos ramos do ano e não afetaram os teores de carboidratos redutores e os totais de aminoácidos e proteínas.

Termos de Indexação: Vitis vinifera, adubação foliar, amido, aminoácidos, proteínas.

\section{NITROGEN FOLIAR SPRAYING IN GRAPEVINE: CONTENT IN LEAVES AND RESERVE OF NITROGEN AND CARBOIHYDRATES IN SHOOTS BUDS}

ABSTRACT - Leaf nitrogen application is used in grapevines in Southern Brazil as complement to soil fertilization. On the other hand, there is no information about its affects on nitrogen content in the leaves and nitrogen and carbohydrates reserves in the perennial parts. The experiment was carried out in 2004/2005, with the objective to evaluate the effect of nitrogen foliar spraying on leaves and nitrogen and carbohydrates reserves in shoots buds of Chenin Blanc grapevine, at Udorthent soil, in Bento Gonçalves, Rio Grande do Sul, Brazil. The treatment was three foliar applications: 0 (water); $1.11 ; 2.23 ; 3.31 ; 4.41 \mathrm{~g} \mathrm{~N}$ grapevine ${ }^{-1}$. Leaves were picked, dried and prepared to total nitrogen analyses. In the last leaf sampling, three shoots and six buds were taken in each plant, prepared and analyzed for starch, total soluble carbohydrates, carbohydrates reducers, total amino acids and total proteins. Leaf nitrogen application increased nitrogen content in the leaf and decreased starch content and total soluble carbohydrates in shoots buds, but did not affect carbohydrates reducers and totals of aminoacids and proteins.

Index terms: Vitis Vinifera, foliar fertilization, starch, aminoacids, proteins.

\section{INTRODUÇÃOO}

Na maior região vitícola do Brasil, a Serra Gaúcha do Rio Grande do Sul (RS), as videiras anualmente são submetidas à aplicação de nitrogênio $(\mathrm{N})$ via solo. Entretanto, sua recuperação e acumulação são pequenas (Brunetto, 2004; Brunetto et al., 2006b) e, por isso, pode ser necessária a complementação de $\mathrm{N}$ via aplicação foliar (Tagliavini \& Toselli, 2005). As folhas da videira apresentam cutícula pouco espessa, grande espaço intercelular do tecido lacunoso e abundância de pêlos na superfície adaxial (Fregoni, 1980), o que facilita a absorção do N aplicado. A aplicação foliar de $\mathrm{N}$ é uma prática comum durante o período vegetativo e produtivo da videira. Após a colheita, as folhas ainda estão fotossinteticamente ativas, assim a absorção de $\mathrm{N}$ pode aumentar a quantidade de reservas nitrogenadas para a safra posterior.

1(Trabalho 274-07). Recebido em:21-11-2007. Aceito para publicação em: 02-07-2008. Trabalho realizado com recursos da Embrapa Uva e Vinho. ${ }^{2}$ Engenheiro Agrônomo, Doutor em Ciência do Solo, Pós-Doutorando do Programa de Pós-Graduação em Ciência do Solo da Universidade Federal de Santa Maria (UFSM). Bolsista da Capes. Rua Erly de Almeida Lima, 546, Apartamento 302, Bairro Camobi, Cep: 97105-120, Santa Maria-RS, Brasil. Email: brunetto.gustavo@gmail.com (autor para correspondência).

${ }^{3}$ Engenheiro Agrônomo, Doutorando do Programa de Pós-Graduação em Ciência do Solo da UFSM. UFSM, Centro de Ciências Rurais (CCR), Dep. de Solos, Caixa Postal 221, Cep: 97105-900, Santa Maria-RS, Brasil. E-mail: eduardogirotto@ hotmail.com

${ }^{4}$ Engenheiro Agrônomo, Doutor em Ciência do Solo, Pesquisador da Embrapa Uva e Vinho. Embrapa Uva e Vinho, Caixa Postal 130, Rua Livramento 515, Cep: 95700-000, Bento Gonçalves-RS, Brasil. E-mail: george@cnpuv.embrapa.br, henrique@cnpuv.embrapa.br

${ }_{5}^{5}$ Engenheiro Agrônomo, Doutor em Ciência do Solo, Professor do Departamento de Solos da Universidade Federal de Santa Maria (UFSM). Bolsista em produtividade do CNPq. UFSM, Centro de Ciências Rurais (CCR), Dep. de Solos, Caixa Postal 221, Cep: 97105-900, Santa Maria-RS, Brasil. E-mail: carlosceretta@smail.ufsm.br, jk@smail.ufsm.br

${ }^{6}$ Acadêmico do Curso de Agronomia da Universidade Federal de Santa Maria (UFSM). Bolsista de Iniciação Científica do CNPq. UFSM, Centro de Ciências Rurais (CCR), Dep. de Solos, Caixa Postal 221, Cep: 97105-900, Santa Maria-RS, Brasil. E-mail: renancbvieira@yahoo.com.br 
Estudos em frutíferas têm mostrado que o $\mathrm{N}$ aplicado via foliar aumenta o seu teor interno (Sanches et al., 1990; Fallahi et al., 2002), sendo incorporado aos esqueletos carbônicos, formando compostos de baixa relação $\mathrm{C} / \mathrm{N}$, o que estimula a degradação das reservas de carbono nas partes perenes, entre as quais o amido, sendo que o carbono resultante é usado como fonte de energia e estrutura na síntese de proteínas e aminoácidos (Yoshioka et al., 1988; Guak \& Fuchigami, 2002; Chen \& Cheng, 2003; Cheng et al., 2004; Xia \& Cheng, 2004). Esses compostos são redistribuídos para as partes perenes (Kliewer \& Cook, 1974; Brunetto, 2004; Brunetto et al., 2005) e podem ser remobilizados e usados para o crescimento de tecidos jovens no ciclo subseqüente. Porém, aumentos do conteúdo de $\mathrm{N}$ na folha e das reservas nas partes perenes da videira estão associados à dose do nutriente aplicada e, por isso, torna-se necessária a realização de experimentos de campo para estimar o efeito dessas aplicações, especialmente quando usado na forma de uréia.

O trabalho objetivou avaliar o efeito de aplicações foliares de nitrogênio no seu teor nas folhas e sobre suas reservas e de carboidratos nas gemas dos ramos do ano.

\section{MATERIAL E MÉTODOS}

O trabalho foi conduzido na área experimental da Embrapa Uva e Vinho, no município de Bento Gonçalves (RS), (Latitude $29^{\circ} 09^{\prime} 44^{\prime \prime} \mathrm{S}$ e Longitude $51^{\circ} 31^{\prime} 50^{\prime \prime} \mathrm{W}$ ), safra 2004/05. O experimento foi instalado em um vinhedo de viníferas, cultivar Chenin Blanc, enxertada sobre o porta-enxerto 101-14. As videiras foram plantadas no ano de 1986, com espaçamento de 1,5m entre plantas e 2,5m entre fileiras e conduzidas em espaldeira. O solo do experimento foi um Neossolo Litólico (Embrapa, 1999) com os seguintes atributos: argila $343 \mathrm{~g} \mathrm{~kg}^{-1}$; silte $357 \mathrm{~g} \mathrm{~kg}^{-1}$; areia $300 \mathrm{~g}$ $\mathrm{kg}^{-1}$; matéria orgânica $26 \mathrm{~g} \mathrm{~kg}^{-1} ; \mathrm{pH}$ em água 5,8; Índice SMP 5,9; Ca trocável $91,9 \mathrm{mmol}_{\mathrm{c}} \mathrm{dm}^{-3} ; \mathrm{Mg}$ trocável $19,8 \mathrm{mmol}_{\mathrm{c}} \mathrm{dm}^{-3} ; \mathrm{Al}$ trocável 0,0mmol $\mathrm{dm}^{-3} ; \mathrm{P}$ disponível (Mehlich-1) 30,6 $\mathrm{mg} \mathrm{dm}^{-3} \mathrm{e} \mathrm{K}$ trocável $4,0 \mathrm{mmol}_{\mathrm{c}} \mathrm{dm}^{-3}$. Os dados climáticos durante a condução do experimento são apresentados na Tabela 1.

As videiras foram submetidas a uma aplicação foliar de 0 (água); 1,$11 ; 2,23 ; 3,31$ e 4,41g de $\mathrm{N}$ planta $^{-1}$, equivalente a 0 ; 2,$97 ; 5,94 ; 8,82$ e $11,76 \mathrm{~kg} \mathrm{ha}^{-1}$ de $\mathrm{N}$, a duas aplicações, correspondente a $0 ; 5,94 ; 11,88 ; 17,64$ e $23,52 \mathrm{~kg} \mathrm{ha}^{-1}$ de $\mathrm{N}$ e a três aplicações, equivalente a $0 ; 8,91 ; 17,82 ; 26,46$ e $35,28 \mathrm{~kg} \mathrm{ha}^{-1}$ de $\mathrm{N}$. A primeira aplicação de $\mathrm{N}$ foi realizada aos sete dias antes da colheita da uva (28-01-04), a segunda um dia depois da colheita da uva (04-02-04) e a terceira sete dias após a segunda aplicação (11-02-04). A fonte de $\mathrm{N}$ foi uréia, diluída em água e aplicado $300 \mathrm{~mL}$ da solução em cada planta, aproximadamente, $800 \mathrm{~L} \mathrm{ha}^{-1}$. Todas as aplicações foram realizadas usando pulverizador manual e no intervalo entre oito e dez horas da manhã. O delineamento experimental usado foi o de blocos ao acaso, com três repetições e três plantas por parcela, que foram distribuídas ao longo da linha de plantio.

Nas videiras submetidas a uma aplicação foliar de $\mathrm{N}$, foram coletadas folhas completas (limbo+pecíolo) do terço médio dos ramos do ano, nos diferentes lados da planta, de acordo com o procedimento descrito pela CFS-RS/SC (1994) às 3 horas e 7; 14; 21; 28 e 35 dias depois da aplicação. Nas videiras submetidas a duas aplicações de $\mathrm{N}$, foram coletadas folhas completas às 3 horas e 7; 14; 21 e 28 dias depois da aplicação. Nas videiras onde foram feitas três aplicações foliares de N, as folhas completas foram coletadas às 3 horas e 7; 14 e 21 dias depois da aplicação.

As folhas coletadas foram secas, moídas e preparadas para a análise de $\mathrm{N}$ total, conforme metodologia proposta por Tedesco et al. (1995). Na última época de coleta de folhas, em todas as aplicações de $\mathrm{N}$, foram coletados três ramos do ano em cada planta. Em seguida, foram retiradas seis gemas em cada ramo, duas na base, duas na parte média e duas no ápice. Posteriormente, as gemas foram congeladas em freezer, à temperatura de $-15^{\circ} \mathrm{C}$ até o momento das análises de reserva. As amostras de gemas foram separadas em duas frações, sendo uma fração mantida congelada e a outra seca em estufa $\left(70^{\circ} \mathrm{C}\right)$ até massa constante. A fração seca foi triturada, sendo três subfrações de $100 \mathrm{mg}$ de cada amostra submetidas a quatro extrações $\left(100 \mathrm{~mL} \mathrm{mg}^{-1}\right)$ com etanol $80 \%$, por 30 minutos. Os sobrenadantes das extrações foram submetidos às análises espectrofotométricas de açúcares solúveis totais (Dubois et al., 1956) e açúcares redutores (Nelson, 1944). Os precipitados das extrações alcoólicas foram secos em temperatura ambiente e submetidos à análise de amido, pelo método enzimático descrito por Arêas \& Lajolo (1980), seguindo modificações citadas por Santos et al. (2004). A fração das gemas que foi mantida intacta e congelada, foi submetida a uma trituração, com auxílio de nitrogênio líquido, e 6 subfrações de 500mg de cada amostra foram separadas para análises de aminoácidos totais (3 subamostras) e proteínas totais (3 subamostras), conforme metodologias descritas em Passos (1996).

Os resultados obtidos foram submetidos à análise de variância e, quando os efeitos foram significativos, foram ajustadas equações de regressão, testando-se os modelos linear e quadrático pelo teste $\mathrm{F}$, escolhendo-se aquele com significância maior que $95 \%$.

\section{RESULTADOS E DISCUSSÃO}

A aplicação das doses de $\mathrm{N}$ apenas aos sete dias antes da colheita da uva promoveu um aumento linear na concentração de $\mathrm{N}$ nas folhas coletadas aos sete e 14 dias após a aplicação (Tabela 2). Com duas aplicações, o aumento do $\mathrm{N}$ na folha aconteceu até os 21 dias. Por outro lado, com três aplicações, a concentração na folha aumentou somente até sete dias após a aplicação. Esses resultados mostram que a concentração de N na folha inteira aumenta com a dose do nutriente aplicada e são mais altas nas épocas de avaliação próxima à aplicação, como foi observado por Sanches et al. (1990) em pereira e por Fallahi et al. (2002) em macieira. Isso pode acontecer porque, em geral, as frutíferas, entre as quais a videira, possuem folhas com cutícula pouco espessa, grandes espaços intercelulares do tecido lacunoso e abundância de pêlos na superfície adaxial (Fregoni, 1980), o que facilita a retenção e posterior absorção do N aplicado nas primeiras horas depois da sua aplicação (Rosecrance et al., 1998; Orbovic et al., 2001; Baldi et al., 2004). Os resultados 
sugerem que uma parte do $\mathrm{N}$ aplicado pode ter sido redistribuído para outros tecidos das plantas, como os ramos do ano, do ano anterior e caule, como relatado por Brunetto (2004) e Brunetto et al. (2005). No entanto, como não se verificou aumento de reservas nitrogenadas, como os aminoácidos e proteínas, nas gemas dos ramos do ano, acredita-se que parte do $\mathrm{N}$ aplicado pode ter sido transferido para a atmosfera na forma de amônia, como reportado por Orbovic et al. (2001), e ainda o $\mathrm{N}$ remanescente na parte externa da folha pode ter sido lavado com a água da chuva, especialmente em meses com precipitações freqüentes, como observado em fevereiro e março de 2004 (Tabela 1).

Os teores de carboidratos não-estruturais, representados pelos valores de amido e de carboidratos solúveis totais, diminuíram nas gemas dos ramos do ano em todas as doses de $\mathrm{N}$ e aplicações (Tabela 3), o que pode indicar que esses compostos foram utilizados como esqueletos carbônicos para a incorporação do $\mathrm{N}$ orgânico. Apesar de significativa, a diminuição nessas reservas de carbono não foi tão expressiva, concordando com os dados obtidos por Chen \& Cheng (2003) e Xia \& Cheng (2004), em experimentos com videiras jovens. Cabe ressaltar que os menores valores de amido nas gemas dos ramos do ano foram encontrados na dose de $2,23 \mathrm{~g} \mathrm{~N}$ planta $^{-1}$, quando o $\mathrm{N}$ foi aplicado em uma única vez e na dose de $1,11 \mathrm{~g} \mathrm{~N}$ planta $^{-1}$, quando o $\mathrm{N}$ foi aplicado duas e três vezes (Tabela 3 ).

Nas doses de 1,11 e 3,31 $\mathrm{g} \mathrm{N}_{\text {planta }^{-1}}$, em todas as aplicações, os valores de amido nas gemas dos ramos do ano foram menores, comparativamente às demais doses do nutriente aplicadas (Tabela 3). Esses resultados, possivelmente, se devem ao fato de que a aplicação de $\mathrm{N}$ em um período de senescência das folhas estimula a degradação de reservas de carbono acumuladas em partes perenes durante o ciclo vegetativoprodutivo, sendo esse carbono usado como fonte de energia e estrutura na síntese de compostos orgânicos nitrogenados, como observado por Yoshioka et al. (1988), Guak \& Fuchigami (2002), Chen \& Cheng (2003), Cheng et al. (2004) e Xia \& Cheng (2004). Entretanto, convém salientar que, na maior dose $\left(4,41 \mathrm{~g} \mathrm{~N}\right.$ planta $\left.^{-1}\right)$, em todas as aplicações, os valores de amido foram maiores. Isso pode ser atribuído a um possível efeito inibitório do $\mathrm{N}$, em concentrações maiores, sobre a atividade da enzima alfaamilase. Isso porque, estudos com essa enzima, isolada de fungos, mostram que a sua atividade é inibida completamente em meio onde as concentrações de $\mathrm{N}$ no substrato ultrapassam $8 \mathrm{mmol} \mathrm{L}$ ${ }^{1}$ (Burhan et al., 2003; Wu et al., 2007). Esse efeito é mais evidente quando se observa que os teores de amido e açúcares solúveis totais diminuíram, possivelmente, em decorrência da limitação no processo de degradação das reservas de amido. Por outro lado, quando as doses de $\mathrm{N}$ foram distribuídas em três aplicações, verifica-se que o dreno pelos carboidratos de reserva foi mais expressivo, mesmo em menores dosagens (Tabela 3). Esse avanço na senescência foliar pela aplicação de $\mathrm{N}$ pode diminuir o período de atividade fotossintética, assemelhando-se aos dados obtidos por Xia \& Cheng (2004) em videiras da cultivar Concord.

Apesar dos efeitos da aplicação de $\mathrm{N}$ sobre as reservas de carbono, destaca-se que as doses de $\mathrm{N}$ aplicadas em uma, duas e três vezes não afetaram o acúmulo de aminoácidos e proteínas (Tabela 3) nas gemas dos ramos do ano. De acordo com Xia \& Cheng (2004), aproximadamente $60 \%$ do carbono que é mobilizado das reservas, é convertido em proteínas e aminoácidos, e o restante é consumido pelo metabolismo respiratório. Com isso, pode inferir-se que a absorção/ metabolização do $\mathrm{N}$ aplicado via foliar no final do ciclo anual pode ter sido de pouca importância, não alterando a síntese de novos aminoácidos e proteínas nos ramos do ano. Essa baixa resposta dos níveis de aminoácidos e proteínas à aplicação foliar de $\mathrm{N}$ está associada à disponibilidade desse nutriente no solo e à sua absorção pela planta durante o ciclo vegetativo e produtivo (Guak \& Fuchigami, 2002). Assim, o solo deste experimento pode ter fornecido uma quantidade satisfatória de $\mathrm{N}$ para as videiras, como relatado por Brunetto (2004), Brunetto et al. (2006a) e Brunetto et al. (2006b), sendo de pouca importância a aplicação foliar de $\mathrm{N}$ para o aumento dos valores de aminoácidos e proteínas nos ramos do ano.

TABELA 1 - Características climáticas da área experimental da Embrapa Uva e Vinho, município de Bento Gonçalves (RS), durante os meses do ano de 2004.

\begin{tabular}{lccccc}
\hline Mês & $\begin{array}{c}\text { Temperatura } \\
\text { do } \operatorname{ar}\left({ }^{\circ} \mathrm{C}\right)\end{array}$ & $\begin{array}{c}\text { Precipitação } \\
\text { Pluviométrica }(\mathrm{mm})\end{array}$ & $\begin{array}{c}\mathrm{N}^{\mathrm{o}} \text { dias com } \\
\text { precipitação }\end{array}$ & $\begin{array}{c}\text { Umidade } \\
\text { relativa do ar }(\%)\end{array}$ & $\begin{array}{c}\text { Insolação } \\
(\text { horas })\end{array}$ \\
\hline & & & & & 74 \\
Janeiro & 21,8 & 97 & 8 & 74 & 270 \\
Fevereiro & 20,5 & 134 & 8 & 251 & 246 \\
Março & 20,2 & 53 & 84 & \\
\hline
\end{tabular}


TABELA 2 - Teor de nitrogênio total nas folhas de videiras, cultivar Chenin Blanc, submetidas a aplicações foliares de nitrogênio.

\begin{tabular}{|c|c|c|c|c|c|c|c|c|}
\hline \multirow{2}{*}{ Nitrogênio } & \multicolumn{6}{|c|}{ Época de coleta das folhas após a aplicação de nitrogênio } & \multirow{2}{*}{ Equação de regressão } & \multirow{2}{*}{$\mathrm{R}^{2}$} \\
\hline & 3 horas & 7 dias & 14 dias & 21 dias & 28 dias & 35 dias & & \\
\hline g planta $^{-1}$ & \multicolumn{8}{|c|}{$\begin{array}{l}\text { Nitrogênio na folha inteira, \% } \\
\text { Uma aplicação foliar de N }\end{array}$} \\
\hline 0 & $2,61^{1}$ & $2,18^{2}$ & $2,27^{3}$ & $2,36^{\mathrm{ns}}$ & $2,23^{\mathrm{ns}}$ & $1,91^{\text {ns }}$ & $y=2,505-0,0136 x$ & $0,53^{*}$ \\
\hline 1,11 & 2,87 & 2,38 & 2,71 & 2,58 & 2,51 & 2,00 & $y=2,821-0,0174 x$ & $0,51^{*}$ \\
\hline 2,23 & 2,93 & 2,30 & 2,53 & 2,33 & 2,33 & 1,88 & $y=2,788-0,0225 x$ & $0,66^{*}$ \\
\hline 3,31 & 2,77 & 2,27 & 2,64 & 2,54 & 2,62 & 1,93 & $y=2,709-0,0138 x$ & $0,30^{*}$ \\
\hline 4,41 & 3,21 & 2,36 & 2,65 & 2,58 & 2,50 & 2,02 & $y=2,970-0,0230 x$ & $0,53^{*}$ \\
\hline \multirow[t]{2}{*}{$\mathrm{CV}, \%$} & 10,84 & 2,67 & 5,70 & 5,82 & 10,16 & 4,14 & & \\
\hline & \multicolumn{8}{|c|}{ Duas aplicações foliares de $\mathrm{N}$} \\
\hline 0 & $2,18^{4}$ & $2,20^{5}$ & $2,36^{6}$ & $2,23^{7}$ & $1,86^{\mathrm{ns}}$ & - & $y=2,315-0,0010 x$ & $0,32^{*}$ \\
\hline 1,11 & 2,80 & 2,58 & 2,59 & 2,52 & 1,85 & - & $y=2,917-0,0308 x$ & $0,75^{*}$ \\
\hline 2,23 & 3,26 & 2,74 & 2,48 & 2,45 & 1,96 & - & $y=3,220-0,0440 x$ & $0,88^{*}$ \\
\hline 3,31 & 3,26 & 2,77 & 2,64 & 2,38 & 1,95 & - & $y=3,274-0,0462 x$ & $0,94^{*}$ \\
\hline 4,41 & 3,34 & 2,76 & 2,65 & 2,56 & 1,78 & - & $y=3,362-0,0510 x$ & $0,86^{*}$ \\
\hline $\mathrm{CV}, \%$ & 5,94 & 5,14 & 5,89 & 5,39 & 5,32 & & & \\
\hline \multicolumn{9}{|c|}{ Três aplicações foliares de $\mathrm{N}$} \\
\hline 0 & $2,20^{8}$ & $2,36^{9}$ & $2,23^{\mathrm{ns}}$ & $1,86^{\text {ns }}$ & - & - & $y=2,395-0,0207 x$ & $0,59^{*}$ \\
\hline 1,11 & 2,77 & 2,56 & 2,26 & 1,93 & - & - & $y=2,898-0,0461 x$ & $0,99^{*}$ \\
\hline 2,23 & 3,00 & 2,63 & 2,35 & 2,00 & - & - & $y=3,088-0,0528 x$ & $0,97^{*}$ \\
\hline 3,31 & 3,22 & 2,55 & 2,42 & 1,66 & - & - & $y=3,330-0,0772 x$ & $0,91^{*}$ \\
\hline 4,41 & 3,47 & 2,52 & 2,45 & 2,00 & - & - & $y=3,339-0,0694 x$ & $0,79^{*}$ \\
\hline $\mathrm{CV}, \%$ & 7,66 & 7,26 & 6,25 & 4,49 & & & & \\
\hline
\end{tabular}

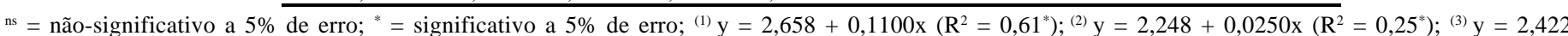
$+0,0690 \mathrm{x}\left(\mathrm{R}^{2}=0,39^{*}\right) ;{ }^{(4)} \mathrm{y}=2,412+0,2780 \mathrm{x}\left(\mathrm{R}^{2}=0,80^{*}\right) ;{ }^{(5)} \mathrm{y}=2,348+0,1310 \mathrm{x}\left(\mathrm{R}^{2}=0,73^{*}\right) ;{ }^{(6)} \mathrm{y}=2,418+0,0630 \mathrm{x}\left(\mathrm{R}^{2}=0,65^{*}\right) ;{ }^{(7)} \mathrm{y}=2,324+0,0520 \mathrm{x}$ $\left(\mathrm{R}^{2}=0,39^{*}\right) ;{ }^{(8)} \mathrm{y}=2,334+0,2990 \mathrm{x}\left(\mathrm{R}^{2}=0,95^{*}\right) ;{ }^{(9)} \mathrm{y}=2,462+0,0310 \mathrm{x}\left(\mathrm{R}^{2}=0,24^{*}\right)$.

TABELA 3 - Teor de amido, carboidratos solúveis totais, carboidratos redutores, aminoácidos totais e proteínas totais em gemas de ramos do ano, em videiras, cultivar Chenin Blanc, submetidas a aplicações foliares de nitrogênio.

\begin{tabular}{|c|c|c|c|c|c|}
\hline Nitrogênio & Amido & $\begin{array}{l}\text { Carboidratos } \\
\text { solúveis totais }\end{array}$ & $\begin{array}{c}\text { Carboidratos } \\
\text { redutores }\end{array}$ & $\begin{array}{c}\text { Aminoácidos } \\
\text { totais }\end{array}$ & $\begin{array}{c}\text { Proteínas } \\
\text { totais }\end{array}$ \\
\hline g planta $^{-1}$ & & $\overline{\mathrm{mg} 100 \mathrm{mg} \mathrm{MS}}$ & & $\mathrm{mg} 500 \mathrm{mg} \mathrm{MS}$ & $\overline{\mathrm{mg} 100 \mathrm{mg} \mathrm{MS}}$ \\
\hline & \multicolumn{5}{|c|}{ Uma aplicação foliar de N } \\
\hline 0 & $6,62^{1}$ & $3,76^{2}$ & $2,41^{\mathrm{ns}}$ & $0,64^{\mathrm{ns}}$ & $1,19^{\text {ns }}$ \\
\hline 1,11 & 5,20 & 3,47 & 2,48 & 0,67 & 1,17 \\
\hline 2,23 & 4,36 & 3,28 & 2,34 & 0,63 & 1,16 \\
\hline 3,31 & 5,48 & 2,69 & 2,67 & 0,74 & 1,20 \\
\hline 4,41 & 5,99 & 2,71 & 2,46 & 0,72 & 1,19 \\
\hline \multirow[t]{2}{*}{$\mathrm{CV}, \%$} & 16,19 & 23,60 & 14,28 & 15,45 & 5,92 \\
\hline & \multicolumn{5}{|c|}{ Duas aplicações foliares de $\mathrm{N}$} \\
\hline 0 & $6,62^{3}$ & $3,54^{4}$ & $2,41^{\text {ns }}$ & $0,64^{\mathrm{ns}}$ & $1,19^{\text {ns }}$ \\
\hline 1,11 & 4,36 & 3,28 & 2,54 & 0,69 & 1,15 \\
\hline 2,23 & 4,98 & 3,11 & 2,36 & 0,68 & 1,18 \\
\hline 3,31 & 5,56 & 2,71 & 2,33 & 0,65 & 1,20 \\
\hline 4,41 & 5,75 & 2,77 & 2,51 & 0,69 & 1,17 \\
\hline \multirow[t]{2}{*}{$\mathrm{CV}, \%$} & 16,86 & 19,34 & 11,73 & 11,82 & 4,92 \\
\hline & \multicolumn{5}{|c|}{ Três aplicações foliares de $\mathrm{N}$} \\
\hline 0 & $6,62^{5}$ & $2,41^{6}$ & $3,54^{\mathrm{ns}}$ & $0,64^{\text {ns }}$ & $1,19^{\text {ns }}$ \\
\hline 1,11 & 4,61 & 2,54 & 3,52 & 0,61 & 1,17 \\
\hline 2,23 & 4,83 & 2,23 & 2,89 & 0,68 & 1,19 \\
\hline 3,31 & 4,71 & 2,54 & 2,47 & 0,70 & 1,21 \\
\hline 4,41 & 6,13 & 2,60 & 2,71 & 0,69 & 1,22 \\
\hline $\mathrm{CV}, \%$ & 19,95 & 21,21 & 10,94 & 14,91 & 5,44 \\
\hline
\end{tabular}

ns = não-significativo a $5 \%$ de erro; ${ }^{*}=$ significativo a 5\% de erro; ${ }^{(1)} \mathrm{y}=6,557-1,7609 \mathrm{x}+0,4157 \mathrm{x}^{2}\left(\mathrm{R}^{2}=0,87^{*}\right) ;{ }^{(2)} \mathrm{y}=3,758-0,288 \mathrm{x}\left(\mathrm{R}^{2}=0,93^{*}\right) ;{ }^{(3)} \mathrm{y}$ $=6,256-1,4426 \mathrm{x}+0,34710 \mathrm{x}^{2}\left(\mathrm{R}^{2}=0,59^{*}\right) ;{ }^{(4)} \mathrm{y}=3,504-0,2110 \mathrm{x}\left(\mathrm{R}^{2}=0,92^{*}\right) ;{ }^{(5)} \mathrm{y}=6,487-1,9509 \mathrm{x}+0,46570 \mathrm{x}^{2}\left(\mathrm{R}^{2}=0,90^{*}\right) ;{ }^{(6)} \mathrm{y}=2,456-0,0991 \mathrm{x}$ $+0,03430 x^{2}\left(R^{2}=0,35^{*}\right)$.

\section{CONCLUSÃO}

Aplicações foliares de nitrogênio promovem aumento no teor de nitrogênio na folha inteira, apenas por curtos períodos de tempo após a aplicação e podem causar diminuição nos teores de amido e carboidratos solúveis totais nas gemas dos ramos do ano, e não afetar os teores de carboidratos redutores e os totais de aminoácidos e proteínas.

\section{AGRADECIMENTOS}

Aos laboratoristas, Volmir Scanagatta e Alexandre Mussnich (Laboratório de Análise de Solo e Tecido da Embrapa Uva e Vinho), pelo auxílio nas análises laboratoriais.

\section{REFERENCIAS}

ARÊAS, J. A. G.; LAJOLO, F. M. Determinação enzimática específica de amido, glicose, frutose e sacarose em bananas préclimatéricas e climatéricas. In: Anais de Farmácia e Química de São Paulo, São Paulo, v.20, p. 307-318, 1980.

BALDI, E.; TOSELLI, M.; SCUDELLARI, D.; TAGLIAVINI, M.; MARANGONI, B. La concimazione fogliare delle drupacee. L'Informatore Agrario, Verona, v. 21, p. 43-46, 2004.

BRUNETTO, G. Absorção e redistribuição do nitrogênio aplicado em plantas de videira. 2004. 74 f. Dissertação (Mestrado em Ciência do Solo). Universidade Federal de Santa Maria, Santa Maria, 2004. 
BRUNETTO, G.; KAMINSKI, J.; MELO, G. W. B.; BRUNING, F. S.; MALLMANN, F. Destino do nitrogênio em videiras 'Chardonnay' e 'Riesiling Renano' quando aplicado no inchamento das gemas. Revista Brasileira de Fruticultura, Jaboticabal, v. 28, p. 497-500, 2006b.

BRUNETTO, G.; KAMINSKI, J.; MELO, G. W. B.; GATIBONI, L. C.; URQUIAGA, S. Absorção e redistribuição do nitrogênio aplicado via foliar em videiras jovens. Revista Brasileira de Fruticultura, Jaboticabal, v.27, p.110-114, 2005.

BRUNETTO, G.; KAMINSKI, J.; MELO, G. W. B.; RHEINHEIMER, D. S. Recuperação e distribuição do nitrogênio fornecido a videiras jovens. Pesquisa Agropecuária Brasileira, Brasília, v. 41, n. 8, p. 1299-1304, 2006a.

BURHAN, A.; NISA, U.; GÖKHAN, C.; ÖMER, C.; ASHABIL, A.; OSMAN, G. Enzymatic properties of a novel thermostable, thermophilic, alkaline and chelator resistant amylase from an alkaliphilic Bacillus sp. isolate ANT-6. Process Biochemistry, Rickmansworth, v. 38, p.1397-1403, 2003.

CHEN, L.; CHENG, L. Carbon assimilation and carbohydrate metabolism of 'Concord' grape (Vitis labrusca L.) leaves in response to nitrogen supply. Journal of the American Society of Horticultural Science, Alexandria, v. 128, p. 754-760, 2003.

CHENG, L.; MA, F. W.; RANWALA, D. Nitrogen storage and its interaction with carbohydrates of young apple trees in response to nitrogen supply. Tree Physiology, Victoria, v. 24, p. 91-98, 2004.

COMISSÃO DE FERTILIDADE DO SOLO - RS/SC. Recomendações de adubação e calagem para os Estados do Rio Grande do Sul e Santa Catarina. 3.ed. Passo Fundo.: Núcleo Regional Sul/EMBRAPA/CNPT, 1994. 224 p.

DUBOIS, M.; GILLES, K. A.; HAMILTON, J. K.; REBERS, P. A.; SMITH, F. Colorimetric method for determination of sugars and related substances. Analytical Chemistry, Washington, v. 28, p. 350-356, 1956.

EMPRESA BRASILEIRA DE PESQUISA AGROPECUÁRIACNPS. Sistema brasileiro de classificação de solos. Brasília: EMBRAPA, 1999. $412 \mathrm{p}$.

FALLAHI, E.; KHEMIRA, H.; RIGHETTI, T. L.; AZARENKO, A. $\mathrm{N}$. Influence of foliar application of urea on tree growth, fruit quality, leaf minerals, and distribution of urea-derived nitrogen in apples. Acta Horticulturae, Wageningen, n.594, p. 603-610, 2002.

FREGONI, M. Nutrizione e fertilizzazione della vite. Bologna: Edagricole, 1980.418 p.

GUAK, S.; FUCHIGAMI, L. H. Foliar applications of urea or ABA affect growth cessation, leaf senescence and abscission, cold acclimation and levels of reserve nitrogen and carbohydrates in nitrogen treated apple nursery plants. Journal of Horticultural Science Biotechnology, Ahsford, v. 77, p. 137-142, 2002.

KLIEWER, W. M.; COOK, J. Arginine levels in grape canes and fruits as indicators of nitrogen status of vineyards. American Journal of Enology and Viticulture, Davis, v.25, p.111-117, 1974.

NELSON, N.A. A photometric adaptation of the Somogyi method for the determination of glucose. Journal of Biological Chemistry, Bethesda, v. 153, p. 375-380, 1944.

ORBOVIC, V.; ACHOR, D.; PETRACEK, P.; SYVERTSEN, J. P. Air temperature, humidity, and leaf age affect penetration of urea through grapefruit leaf cuticles. Journal of the American Society of Horticultural Science, Alexandria, v.126, p. 44-50, 2001.

PASSOS, L. P. Métodos analíticos e laboratoriais em fisiologia vegetal. Coronel Pacheco: EMBRAPA-CNPGL, 1996. 223 p.

ROSECRANCE R. C.; JOHNSON R. S.; WEINBAUM S. A. The effect of timing of post-harvest foliar urea sprays on nitrogen absorption and partitioning in peach and nectarine trees. Journal Horticultural Science Biotechnology, Asford, v. 73, p. 856-861, 1998.

SANCHES, E. E.; RIGHETTI, T. L.; SUGAR, D.; LOMBARD, P.B. Response of "Comoce" pear trees to a postharvest urea spray. Journal Horticultural Science, Asford, v. 65, p. 541-546, 1990.

SANTOS, H. P.; PURGATTO, E.; MERCIER, H.; BUCKERIDGE, M. S. The control of storage xyloglucan mobilization in cotyledons of Hymenaea courbaril. Plant Physiology, Oxford, v. 135, p. 287-299, 2004.

TAGLIAVINI, M.; TOSELLI, M. Foliar applications of nutrients. In: HILLEL, D. Encyclopaedia of Soils in the Environment. Oxford: Elsevier, 2005. p. 53-60.

TEDESCO, M. J.; GIANELLO, C.; BISSANI, C. A.; BOHNEN, H.; VOLKWEISS, S. J. Análise do solo, planta e outros materiais. 2.ed. Porto Alegre: UFRGS, Departamento de Solos, 1995. 174p. (Boletim Técnico, 5).

WU, S.; ZHU, Y.; CAI, Q.; ZENG, K.; GRIMES, C. A. A wireless magnetoelastic alfa-amilase sensor. Sensors and Actuators B, Warrendale, v. 121, p. 476-481, 2007.

XIA, G. H.; CHENG, L. L. Foliar urea application in the fall affects both nitrogen and carbon storage in young 'Concord' grapevines grown under a wide range of nitrogen supply. Journal of the American Society of Horticultural Science, Alexandria, v. 129, p. 653-659, 2004.

YOSHIOKA, H.; NAGAI, K.; AOBA, K.; FUKUMOTO, M. Seasonal changes of carbohydrates metabolism in apple trees. Scientia Horticulturae, Amsterdan, v.36, p.219-227, 1988. 